\title{
ÉtAT ACTUEL DE LA MiSE AU POINT BIBLIOGRAPHIQUE SUR LE NIOBIUM ET SES ALLIAGES
}

\author{
par F. JEANNE, J. DRIOLE, E. BONNIER
}

\begin{abstract}
Résumé. - Le “ Centre d'Information de Thermodynamique Chimique Minérale 》 présente une bibliographie auto-indexée sur le niobium et ses alliages.

Abstract. - The “ Centre d'Information de Thermodynamique Chimique Minérale » presents a self indexed bibliography on niobium and its alloys.
\end{abstract}

Une mise au point de la bibliographie sur le niobium, décidée conjointement par la DGRST et la DRME, a été confiée au Laboratoire de Thermodynamique et de Physico-Chimie Métallurgiques, associé au C. N. R. S. de Grenoble. Mr. Jeanne a été détaché à cet effet dans ce service, en tant que coopérant DRME, pendant la durée de ses obligations militaires.

$\mathrm{Vu}$ le nombre croissant des publications relatives au niobium, à ses alliages ou composés, une étude générale du sujet risquait, par son ampleur, de dépasser nos possibilités. Le premier point donc consisté à fixer des limites raisonnables dans les différents domaines à inventorier et à définir un ordre de priorité parmi les sujets retenus.

Nous avons alors entrepris l'élaboration d'un fichier et commencé son exploitation pratique.

Nous exposerons rapidement les résultats de ce travail et discuterons, sur un exemple concret, du mode de présentation à adopter par la suite.

I. Résultats. - a. TravaIL PRÉLIMINAIRE D'ORIENTATION. - Deux sortes de recherches ont permis de définir les limites préalables de l'étude : nous avons cherché par le truchement du Comité métallurgie de la DGRST à connaître les souhaits d'un certain nombre de laboratoires intéressés par le niobium. Les réponses recueillies ont mis en évidence des domaines présentant un intérêt général. Il s'agit des données thermochimiques et structurales relatives au niobium et à ses alliages ou composés, de leurs propriétés mécaniques, et des problèmes de corrosion et de revêtements.

Simultanément, nous avons parcouru l'ensemble de la littérature concernant le niobium, en examinant particulièrement les différentes monographies et les quelques bibliographies disponibles pour en dégager le caractère exhaustif.

En janvier 1969, deux ouvrages importants sont venus combler en partie le manque de travaux bibliographiques récents dans ces domaines.

Le premier est une monographie de l'AIEA, publiée par O. Kubaschewski sous le titre : «Niobium, physicochemical properties of its compounds and alloys " qui traite, de façon critique des propriétés thermochimiques, diagrammes de phases, stıuctures cristallines et coefficients de diffusion.

Le second est une bibliographie de l'AGARD réalisée par $\mathrm{R}$. Syre et intitulée : «A bibliography of refractory metals », qui couvre un très large domaine de données aussi bien fondamentales que technologiques. (Diagrammes, Couples de diffusion, impuretés, propriétés physiques et mécaniques, corrosion par les gaz ou les métaux, élaboration et mise en forme, revêtements protecteurs...)

L'ensemble de ces deux livres semblait répondre de façon satisfaisante au principal besoin de données bibliographiques sur le niobium. Cependant un examen détaillé de la littérature a montré que ces ouvrages de base pour raient être utilement complétés par une actualisation des données à l'aide de références postérieures à 1966 , et par une extension de certains domaines partiellement explorés. (Diagrammes ternaires par exemple).

Nous avons décidé d'entreprendre ce travail de mise au point en nous fixant à priori le plan d'étude suivant :

1 - Données thermodynamiques.

2 - Diagrammes d'équilibre, structure des phases et composés, pour les systèmes binaires, ternaires et quaternaires.

3 - Diffusion à l'état solide dans le niobium ses alliages et composés.

$$
\begin{aligned}
& 4 \text { - Propriétés physiques. } \\
& 5 \text { - Propriétés mécaniques. } \\
& 6 \text { - Corrosion. } \\
& 7 \text { - Revêtements protecteurs. }
\end{aligned}
$$

b. EtAT ACtuel DE LA Bibliographie. - Nous avons entrepris la constitution d'un fichier codé rassemblant les données bibliographiques sur des cartes à perforation latérale. Le codage choisi permet une classification rapide par auteur, sujet, et éléments d'alliage. Les articles les plus récents (1969 et fin 1968) 
sont extraits par consultation régulière des principales revues de métallurgie, chimie, cristallographie, etc... Les articles antérieurs proviennent de l'exploitation des revues, type « abstracts».

Le fichier comporte actuellement environ 1500 articles répartis dans les domaines cités précédemment. Information a porté en priorité sur les trois premiers chapitres du Plan : Données thermodynamiques, Diagrammes et diffusion. Ce dernier est présenté comme exemple.

II. Publication de la bibliographie. - Le mode de présentation d'une telle bibliographie est un problème délicat. Nous cherchons actuellement à le résoudre et comptons sur les suggestions des participants à cette table ronde pour orienter notre choix.

Il faut souligner en premier lieu la difficulté pratique de présenter un travail constituant un simple additif à des bibliographies précédentes dans la mesure où il réalise à la fois une actualisation des articles et une extension des sujets abordés. Nous avons décidé pour l'instant de reprendre de façon exhaustive toutes les références postérieures à 1950 , en les faisant précéder des références des compilations et ouvrages bibliographiques correspondants.

Par ailleurs, nous ne pouvions entreprendre à grande échelle un travail d'exposition critique et comparative des différentes données. Contraints de présenter une simple liste de références, nous nous sommes efforcés de les classer de façon que leur recherche soit la plus commode possible. Dans l'exem- ple proposé, cette recherche peut se faire par trois voies complémentaires :

1. - Une entrée analytique qui présente les articles selon un plan détaillé en fonction de leur contenu.

2. - Un Index des titres établi automatiquement selon une méthode dérivée du système KWIC.

3. - Un Index des auteurs également automatique.

L'indexation automatique des titres et des auteurs a été réalisée par l'adaptation des programmes mis au point par le Centre d'Information de Thermodynamique Chimique Minérale de Grenoble pour l'édition de son «Index Thermochimique ». Un premier exemplaire expérimental est présenté. On trouvera dans les premières pages les remarques relatives à l'utilisation de cette bibliographie.

L'orientation ultérieure du travail implique une prise de position sur l'utilité réelle d'une telle bibliographie d'une part et sur le mode d'édition choisi d'autre part.

A cet effet nous avons joint aux exemplaires distribués un questionnaire que nous aimerions voir rempli par les participants :

Note des auteurs. - Depuis la tenue de la « Table Ronde 》 au cours de laquelle le présent exposé a été fait les volumes définitifs "Diffusion à l'état solide » et « Diagrammes de phases et structures des systèmes à base de niobium " ont été diffusés par le Centre d'Information de Thermodynamique Chimique Minérale. 